\title{
Correlates of Performances on Computerized Attention Tasks in Patients with Insomnia Disorder
}

\author{
Soohyun Kim', Juhyun Park², Seong min Oh³, Mi hyun Lee, \\ Jeong Eun Jeon ${ }^{3}$, Ha Young Lee ${ }^{3}$, and Yu Jin Lee ${ }^{3}$ \\ 'Department of Neurology, Gangneung Asan Hospital, Gangneung, Korea \\ ${ }^{2}$ Department of Psychology, University at Buffalo, Buffalo, NY, USA \\ ${ }^{3}$ Department of Psychiatry and Center for Sleep and Chronobiology, Seoul National University College of Medicine, Seoul National University Hospital, Seoul, Korea
}

\begin{abstract}
Objective: Although daytime dysfunction is highly correlated with insomnia disorder, the mechanisms underlying this relationship are poorly understood, and previous studies have not produced consistent results. Thus, the present study compared the attention performance of insomnia patients with that of normal controls to investigate the association of various clinical symptoms with attention in insomnia patients. Methods: The present study included 30 patients with insomnia disorder (INS; 24 females and 6 males, mean age: $43.87 \pm 13.73$ years) and 26 healthy controls (HC; 13 females and 13 males, mean age: 37.08 13.40 years) who completed questionnaires about sleep and affect as well as computerized attention tasks and nocturnal polysomnography assessments. Results: Although there were no significant differences in attention performance between the INS and HC groups, correlation analyses revealed that the two groups exhibited different patterns in the relationships of sleep-related symptoms with attention performance. The insomnia patients reported severe difficulties with sleep initiation or frequent negative thoughts related to sleep, and these symptoms were correlated with poorer attention performance. In contrast, the poor sleep disturbance was associated with good attention performance in the HC group. Conclusion: The present results indicated that the attention complaints of insomnia patients may be associated with the failure to maintain attentional effort due to sleep-related psychological symptoms or anxiety rather than objective cognitive impairments.
\end{abstract}

Key Words: Insomnia; Attention performance; Daily function; Maladaptive beliefs

Received: December 14, 2019 Accepted: January 1, 2020

Corresponding author: Yu Jin Lee, MD, PhD, Department of Psychiatry and Center for Sleep and Chronobiology, Seoul National University College of Medicine, Seoul National University Hospital, 101 Daehak-ro, Jongno-gu, Seoul 03080, Korea.

Tel: 82-2-2072-2456, Fax: 82-2-744-7241, E-mail: ewpsyche@snu.ac.kr

(c) This is an Open Access article distributed under the terms of the Creative Commons Attribution Non-Commercial License (https://creativecommons.org/licenses/bynd4.0) which permits unrestricted non-commercial use, distribution, and reproduction in any medium, provided the original work is properly cited.

\section{INTRODUCTION}

Insomnia is characterized by subjective complaints of difficulty initiating or maintaining sleep and/or experiencing non-restorative sleep. Due to such sleep problems, insomnia patients also frequently complain of daytime impairments across various dimensions, including occupational, academic, and/or social situations [1-3]. In fact, daytime dysfunction is included as an essential item in the diagnostic criteria for insomnia. Although daytime dysfunction is highly correlated with insomnia disorder, understanding regarding the mechanisms underlying this relationship is lacking, and previous studies have not produced consistent results.

To date, most studies of cognition in insomnia patients have focused on attention, memory, and executive function. For exam- ple, a recent meta-analysis [4] found that insomnia patients exhibited decreases in perceptual processes, alertness, complex attention, retention, working memory, episodic memory, and problem solving but not in verbal functions, information processing, selective attention, divided attention (DA), sustained attention (SA), procedural memory, verbal fluency, or cognitive flexibility. Although insomnia generally appears to have negative effects on cognitive functioning, the findings of this meta-analysis suggest that previous studies investigating this issue have not found consistent results, even within a single cognitive domain. Furthermore, although the effects of sleep deprivation on cognitive function have been studied for many years, this literature also includes inconsistent findings. For example, in contrast to the expectation that sleep deprivation causes cognitive dysfunction, 
many findings have been negative. One possible theoretical explanation for these negative results is the lapse hypothesis [5], which proposes that sleep loss does not completely damage cognitive function but rather results in moment-to-moment variability in performance due to an unstable awake state. Thus, between lapses of attention, people who experience sleep loss may function as well as they typically would. Accordingly, attention may be a core element underlying daily dysfunction in insomnia patients.

SA is the ability to maintain focus for long periods of time in complex circumstances while experiencing interference. This type of attention is a fundamental aspect of various daily human activities, such as cooking, reading books, and listening to lectures, and is required by almost all people. Previous studies of SA in insomnia patients typically required participants to pay attention for approximately $10 \mathrm{~min}$. The second type of attention, DA, which involves the ability to simultaneously process two or more elements, is important when multitasking, such as talking while driving; thus, it exacts a higher cognitive processing burden. Some studies have revealed significant impairments in the attention performance of insomnia patients, including slower reaction times (RTs) or lower levels of accuracy, whereas others have not [6-9].

Due to these discrepancies regarding attention performance in insomnia patients, it is important to improve the current understanding of this issue by considering the unique maladaptive thoughts of insomnia patients. Patients with chronic insomnia frequently report misperceptions about sleep $[10,11]$, which is one factor by which bad sleep habits are created. For example, insomnia patients might choose to have a less demanding day, which may include skipping exercise or scheduling a nap to compensate for their sleep loss, which can lead to repeatedly disrupted sleep cycles [12]. Insomnia patients also experience excessive worry about sleep and tend to catastrophize the results of sleep loss, which increases the likelihood of a negative mood [13]. Additionally, an epidemiological study of a community-based sample revealed that people with insomnia were 9.82 times more likely to have significant depression and 17.35 times more likely to have anxiety than people without insomnia, even after controlling for confounding factors such as ethnicity and sex [14]. Therefore, the present study investigated how the negative emotional states or maladaptive beliefs of insomnia patients would affect attention.

The present study compared the attention performance of insomnia patients with that of healthy controls to investigate correlations between attention performance and characteristics of insomnia patients, including negative mood, catastrophic worries about sleep, and maladaptive beliefs. It was hypothesized that attention dysfunction in insomnia patients would vary depending on the specific clinical symptoms.

\section{METHODS}

\section{Participants}

All participants were recruited via advertisements at Seoul Na- tional University Hospital and completed the study requirements voluntarily. The present study included 66 participants who were enrolled from two different sleep-related studies that employed identical procedures for the administrations of questionnaires, computerized attention tasks, and nocturnal polysomnography (nPSG) tests. All participants with insomnia met the Diagnostic and Statistical Manual of Mental Disorders, fifth edition (DSM-5) [15] criteria for chronic insomnia. Patients diagnosed with serious neurological or psychiatric disorders following psychiatric interviews during the enrollment period were excluded from the study. All participants completed a one-night nPSG (Profusion PSG3; Compumedics; Abbotsford, VIC, Australia) assessment in a sleep laboratory to evaluate objective sleep characteristics and to rule out other sleep disorders such as sleep apnea or periodic limb movement disorder.

A total of 10 participants were excluded due to missing questionnaire data (2 participants), missing nPSG data (1 participant), the presence of a sleep disorder based on nPSG results [1 participant had an Apnea-Hypopnea Index (AHI) $>15$, and 3 had a Periodic Limb Movements Index (PLMI) $>15$ ], or a score of zero on the SA task (3 participants). Ultimately, 30 patients with insomnia disorder (INS; 24 females and 6 males, mean age: $43.87 \pm 13.73$ years) and 26 healthy controls (HC; 13 females and 13 males, mean age: $37.08 \pm 13.40$ years) were included in the final analyses. The study was approved by the Institutional Review Board of Seoul National University Hospital (IRB No. 1603-073-748, 1806-174-955).

\section{Computerized attention task}

SA and DA were measured using the computerized comprehensive attention task (CAT), which has subsets of tasks that evaluate comprehensive attention and working memory functions. The CAT has been standardized for children and adolescents [16] as well as adults [17].

The SA task assesses the ability to maintain attention under long and repetitious circumstances. Participants are instructed to press a button for every visual stimulus (various shapes presented every $2 \mathrm{~s}$ ) but not for stimuli of a specific shape ('X' shape), which are the targets. The task persists for $10 \mathrm{~min}$ and participants are required to maintain focus during the long series of presentations to be able to successfully inhibit their responses to the target stimuli. The number of commission errors (CE), number of omission errors (OE), and $\mathrm{RT}$ are recorded as behavioral data.

The DA task assesses the ability to simultaneously process multiple demands. This test is a dual one-back task in which two independent sequences are simultaneously presented with visual and auditory stimuli, and participants are required to divide their attention to receive and process two different types of information sources at the same time. Stimuli are presented every $2 \mathrm{~s}$ for $3 \mathrm{~min}$. CE, OE, and RT are recorded as behavioral data.

\section{Questionnaires}

All participants completed several sleep-related questionnaires, including the Epworth Sleepiness Scales (ESS), the Pitts- 
burgh Sleep Quality Index (PSQI), the Dysfunctional Beliefs and Attitudes about Sleep Scale 16 (DBAS-16), and the Insomnia Severity Index (ISI), as well as the Beck Depression Inventory (BDI) and the Beck Anxiety Inventory (BAI). The PSQI assesses sleep quality in terms of sleep latency (SL), sleep duration, sleep disturbance, and daytime dysfunction over a 1-month period; the DBAS- 16 is an abbreviated version of the DBAS and assesses sleep-related beliefs; the ESS assesses daytime sleepiness; and the ISI assesses the degrees of problems in initiating sleep, maintaining sleep or experiencing early awakening, and distress caused by the sleep problem. The HC subjects also completed the ISI questionnaire. The BDI assesses the intensity of depressive symptoms, and the BAI assesses the intensity of anxiety symptoms over the past week.

\section{Statistical analysis}

All behavioral data were automatically calculated by the computerized task program; $\mathrm{CE}, \mathrm{OE}$, and mean RT were recorded. First, group differences were analyzed with multivariate analysis of variance tests, which was performed four times for each categorized variable, i.e., the behavioral results of the SA task, behavioral results of the DA task, questionnaire scores, and nPSG results. Age and gender were included as covariates.

Second, partial correlations between cognitive performance measures (SA and DA tasks, separately) and various individual variables (questionnaire scores and nPSG) were performed after controlling for age and gender. Because the only difference between the groups was a trend toward a significant difference in the mean RT, only RT was used to perform correlation analyses with the other data (i.e., the questionnaires and nPSG). The correlation analyses on each group were performed separately, and all data were analyzed using PASW Statistics for Windows (Version 18.0; SPSS, Inc., Chicago, IL, USA). p-values $<0.05$ were considered to indicate statistical significance.

\section{RESULTS}

\section{Group comparisons}

Both attention tasks showed trends for significant differences between the two groups in mean RT [SA task: $F(1,54)=3.62$, $\mathrm{p}=0.06$; DA task: $\mathrm{F}(1,54)=3.80, \mathrm{p}=0.06$ ] (Table 1 ), with the INS group having longer RTs than the HC group on both the SA and DA tasks. The INS group also had significantly higher scores on the ISI, PSQI, DBAS, BDI, and BAI (all p<0.05) (Table 1). However, there were no significant differences in ESS scores or nPSG measurements (Table 1).

\section{Correlations with cognitive performance}

\section{Questionnaires}

ESS. There were no significant correlations between the mean $\mathrm{RT}$ and ESS score in either group for either attention task.

PSQI. There were no significant correlations between PSQI
Table 1. Results of the multivariate analysis of variance comparing the variables between the INS and $\mathrm{HC}$ groups

\begin{tabular}{|c|c|c|c|}
\hline & INS $(n=30)$ & $\mathrm{HC}(\mathrm{n}=26)$ & $\mathrm{F}$ \\
\hline \multicolumn{4}{|l|}{ Demographic } \\
\hline Age (yr) & $43.87 \pm 13.73$ & $37.08 \pm 13.40$ & \\
\hline Gender (F/M) & $24 / 6$ & $13 / 13$ & \\
\hline \multicolumn{4}{|l|}{ Attention performance } \\
\hline \multicolumn{4}{|l|}{ SA } \\
\hline Number of OE & $0.60 \pm 1.10$ & $1.31 \pm 3.52$ & 2.50 \\
\hline Number of CE & $6.70 \pm 5.87$ & $6.85 \pm 7.50$ & 0.20 \\
\hline Mean RT (ms) & $457.35 \pm 77.10$ & $412.49 \pm 62.73$ & $3.62^{\dagger}$ \\
\hline \multicolumn{4}{|l|}{ DA } \\
\hline Number of OE & $9.00 \pm 7.35$ & $10.77 \pm 10.12$ & 0.97 \\
\hline Number of CE & $4.60 \pm 6.83$ & $3.73 \pm 3.85$ & 0.48 \\
\hline Mean RT (ms) & $838.25 \pm 147.91$ & $733.21 \pm 156.16$ & $3.80^{\dagger}$ \\
\hline \multicolumn{4}{|l|}{ Questionnaire } \\
\hline ESS & $6.77 \pm 4.26$ & $6.85 \pm 3.82$ & 0.20 \\
\hline PSQI & $10.87 \pm 3.73$ & $4.85 \pm 2.07$ & $38.72^{*}$ \\
\hline ISI & $15.83 \pm 7.36$ & $4.42 \pm 3.49$ & $37.58^{*}$ \\
\hline DBAS-16 & $158.03 \pm 37.52$ & $125.31 \pm 29.72$ & $7.65^{*}$ \\
\hline BDI & $12.07 \pm 10.60$ & $3.00 \pm 4.02$ & $13.17^{*}$ \\
\hline BAI & $12.63 \pm 12.18$ & $3.12 \pm 3.89$ & $9.34^{*}$ \\
\hline \multicolumn{4}{|l|}{ Polysomnography } \\
\hline TST (min) & $400.88 \pm 55.98$ & $431.24 \pm 38.45$ & 2.67 \\
\hline SE (\%) & $85.95 \pm 11.38$ & $90.68 \pm 7.01$ & 0.93 \\
\hline WASO (min) & $52.10 \pm 51.13$ & $33.90 \pm 33.62$ & 0.37 \\
\hline$\%$ stage N1 & $12.00 \pm 4.65$ & $11.85 \pm 5.54$ & 0.36 \\
\hline$\%$ stage N2 & $56.49 \pm 9.03$ & $58.01 \pm 9.12$ & 0.71 \\
\hline$\%$ stage N3 & $10.06 \pm 7.73$ & $8.70 \pm 6.00$ & 0.91 \\
\hline$\%$ stage REM & $26.72 \pm 27.43$ & $21.45 \pm 5.88$ & 0.74 \\
\hline $\mathrm{SL}(\min )$ & $12.40 \pm 19.35$ & $10.18 \pm 7.27$ & 0.16 \\
\hline REML (min) & $91.76 \pm 46.36$ & $111.26 \pm 48.07$ & 2.37 \\
\hline AHI (/hr) & $2.86 \pm 2.77$ & $3.56 \pm 4.03$ & 0.24 \\
\hline PLMI (/hr) & $2.36 \pm 3.78$ & $2.10 \pm 3.63$ & 0.03 \\
\hline
\end{tabular}

Data are presented as mean \pm SD. ${ }^{*} \mathrm{p}<0.05,{ }^{\dagger} \mathrm{p}=0.06$. INS: patients with insomnia disorder, HC: healthy control, OE: omission error, CE: commission error, RT: reaction time, SA: sustained attention, DA: divided attention, ESS: Epworth Sleepiness Scales, PSQI: Pittsburgh Sleep Quality Index, ISI: Insomnia Severity Index, DBAS-16: Dysfunctional Beliefs and Attitudes about Sleep Scale 16, BDI: Beck Depression Inventory, BAI: Beck Anxiety Inventory, TST: total sleep time, SE: sleep efficiency, WASO: wake after sleep onset, SL: sleep latency, REML: REM latency, AHI: Apnea-Hypopnea Index, PLMI: Periodic Limb Movement Index

score and mean RT on either attention task in either group. However, trends toward significance were found in the HC group for the associations of mean RT with both attention tasks and with the PSQI score (SA task: $r=-0.37, p=0.07$, DA task: $r=$ $-0.38, \mathrm{p}=0.07$ ) (Table 2). Subsequent correlation analyses assessing the sub-components of the PSQI questionnaire revealed a significant negative correlation between the mean RT on the DA task and PSQI component number 1 (subjective sleep quality; a higher score is indicative of worse quality) in the HC group ( $\mathrm{r}=$ $-0.46, \mathrm{p}<0.05)$ (Fig. 1A).

DBAS-16. There were no significant correlations between the 
Table 2. Results of the correlation analyses between mean reaction time in both attention tasks and the individual variables in both groups

\begin{tabular}{|c|c|c|c|c|}
\hline & \multicolumn{2}{|c|}{ Sustained attention $(\mathrm{r})$} & \multicolumn{2}{|c|}{ Divided attention $(\mathrm{r})$} \\
\hline & $\begin{array}{c}\text { INS } \\
(\mathrm{n}=30)\end{array}$ & $\begin{array}{c}\mathrm{HC} \\
(\mathrm{n}=26)\end{array}$ & $\begin{array}{c}\text { INS } \\
(\mathrm{n}=30)\end{array}$ & $\begin{array}{c}\mathrm{HC} \\
(\mathrm{n}=26)\end{array}$ \\
\hline \multicolumn{5}{|l|}{ Questionnaire } \\
\hline ESS & 0.11 & -0.02 & -0.01 & 0.00 \\
\hline PSQI & 0.17 & -0.37 & 0.18 & -0.38 \\
\hline PSQI component 1 & 0.15 & -0.28 & 0.17 & $-0.46^{*}$ \\
\hline PSQI component 2 & 0.04 & -0.24 & 0.00 & -0.09 \\
\hline PSQI component 3 & 0.03 & -0.06 & 0.14 & -0.22 \\
\hline PSQI component 4 & 0.02 & -0.32 & 0.01 & -0.21 \\
\hline PSQI component 5 & -0.10 & -0.17 & 0.11 & -0.34 \\
\hline PSQI component 6 & 0.33 & -0.16 & 0.30 & -0.09 \\
\hline PSQI component 7 & 0.12 & -0.33 & -0.05 & -0.23 \\
\hline ISI & 0.22 & -0.30 & $0.40^{*}$ & $-0.58^{*}$ \\
\hline ISI la & 0.14 & -0.21 & $0.38^{*}$ & -0.30 \\
\hline ISI $1 b$ & 0.04 & -0.31 & 0.06 & -0.31 \\
\hline ISI $1 \mathrm{c}$ & 0.06 & -0.40 & 0.13 & -0.29 \\
\hline ISI 2 & 0.30 & -0.19 & 0.35 & -0.21 \\
\hline ISI 3 & 0.16 & -0.01 & $0.44^{*}$ & $-0.42^{*}$ \\
\hline ISI 4 & 0.35 & 0.01 & $0.57^{*}$ & -0.35 \\
\hline ISI 5 & 0.22 & -0.25 & $0.44^{*}$ & $-0.47^{*}$ \\
\hline DBAS-16 & -0.24 & 0.09 & 0.16 & 0.12 \\
\hline BDI & 0.01 & -0.18 & 0.29 & -0.32 \\
\hline BAI & -0.09 & -0.25 & $0.39^{*}$ & -0.28 \\
\hline \multicolumn{5}{|l|}{ Polysomnography } \\
\hline TST (min) & 0.04 & 0.38 & -0.17 & 0.30 \\
\hline SE (\%) & -0.03 & $0.42^{*}$ & -0.27 & 0.25 \\
\hline WASO (min) & 0.11 & -0.40 & 0.18 & -0.20 \\
\hline$\%$ stage N1 & 0.13 & 0.11 & 0.22 & -0.26 \\
\hline$\%$ stage N2 & 0.10 & 0.00 & 0.10 & -0.35 \\
\hline$\%$ stage N3 & -0.14 & -0.17 & -0.06 & $0.58^{*}$ \\
\hline \% stage REM & -0.03 & 0.12 & $0.39^{*}$ & 0.11 \\
\hline $\mathrm{SL}(\mathrm{min})$ & 0.04 & -0.19 & $0.44^{*}$ & -0.20 \\
\hline REML (min) & 0.10 & -0.17 & 0.20 & -0.09 \\
\hline AHI (/hr) & -0.15 & -0.06 & 0.01 & -0.11 \\
\hline PLMI (/hr) & -0.19 & -0.09 & -0.33 & -0.15 \\
\hline
\end{tabular}

${ }^{*} \mathrm{p}<0.05$. INS: patients with insomnia disorder, HC: healthy control, ESS: Epworth Sleepiness Scales, PSQI: Pittsburgh Sleep Quality Index, ISI: Insomnia Severity Index, DBAS-16: Dysfunctional Beliefs and Attitudes about Sleep Scale 16, BDI: Beck Depression Inventory, BAI: Beck Anxiety Inventory, TST: total sleep time, SE: sleep efficiency, WASO: wake after sleep onset, SL: sleep latency, REML: REM latency, Apnea-Hypopnea Index, PLMI: Periodic Limb Movement Index

mean RT and DBAS score in either group or on either attention task.

ISI. The two groups exhibited opposite correlation patterns, particularly for the mean RT on the DA task. The INS group showed a positive correlation between ISI score and mean RT on the DA task $(\mathrm{r}=0.40, \mathrm{p}<0.05)$, whereas the $\mathrm{HC}$ group showed a negative correlation $(r=-0.58, p<0.05)$ (Table 2, Fig. 2). In subsequent correlation analyses of the sub-items of the ISI questionnaire, the INS group showed a trend toward a positive correlation for all items

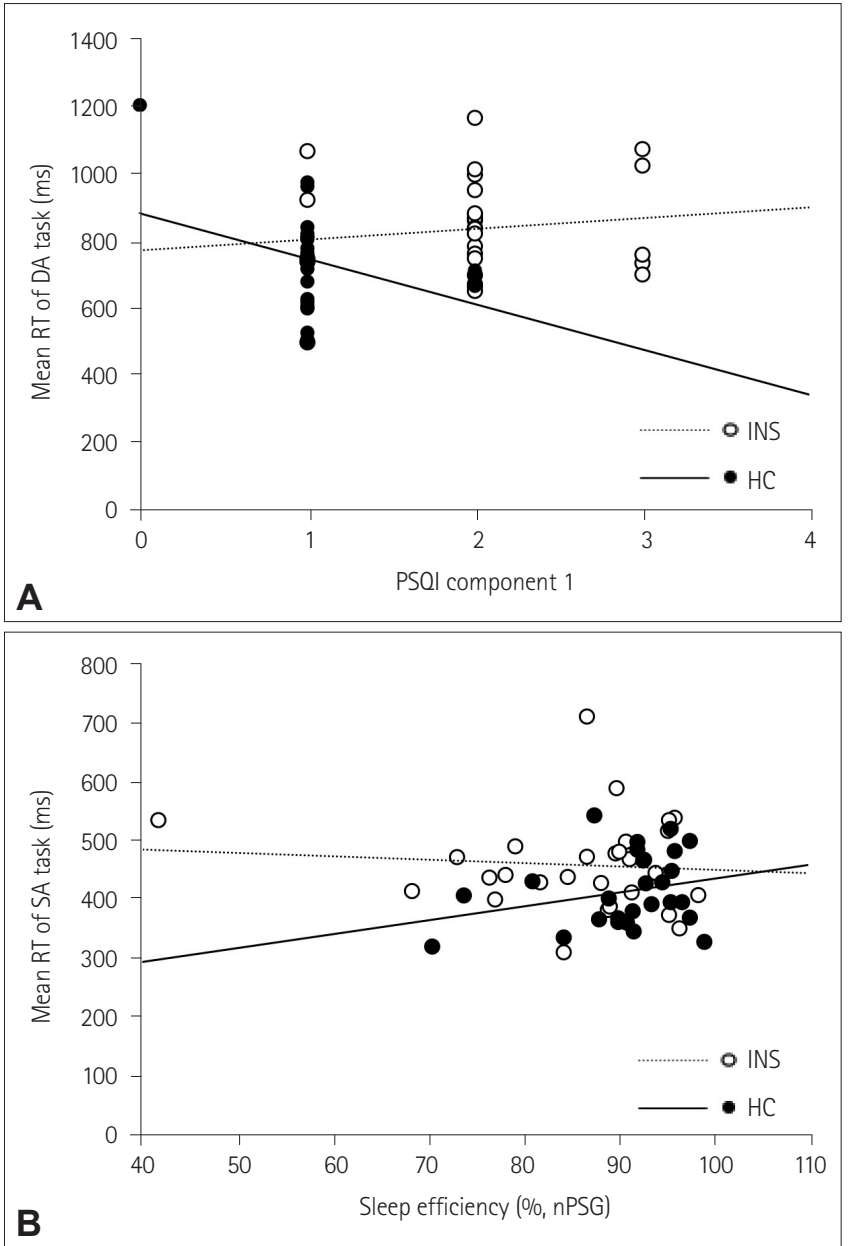

Figure 1. Correlations between attention performances and PSQI sub-item scores. (A) In the HC group, a lower subjective sleep quality (PSQI component 1: a higher score is indicative of worse quality) was associated with better attention performance. (B) A similar correlation between attention performance and sleep efficiency (measured by nPSG) was also observed. PSQI: Pittsburgh Sleep Quality Index, INS: patients with insomnia disorder, HC: healthy control, nPSG: nocturnal polysomnography, $\mathrm{RT}$ : reaction time, DA: divided attention, SA: sustained attention.

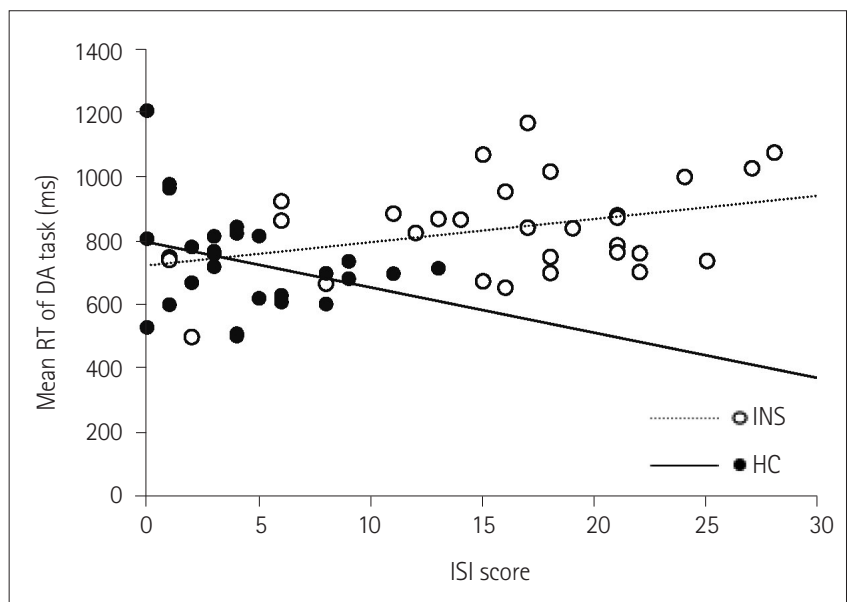

Figure 2. Correlation between DA performance and ISI score. Insomnia patients with a higher ISI score had poorer attention performance. ISI: Insomnia Severity Index, INS: patients with insomnia disorder, HC: healthy control, RT: reaction time, DA: divided attention. 

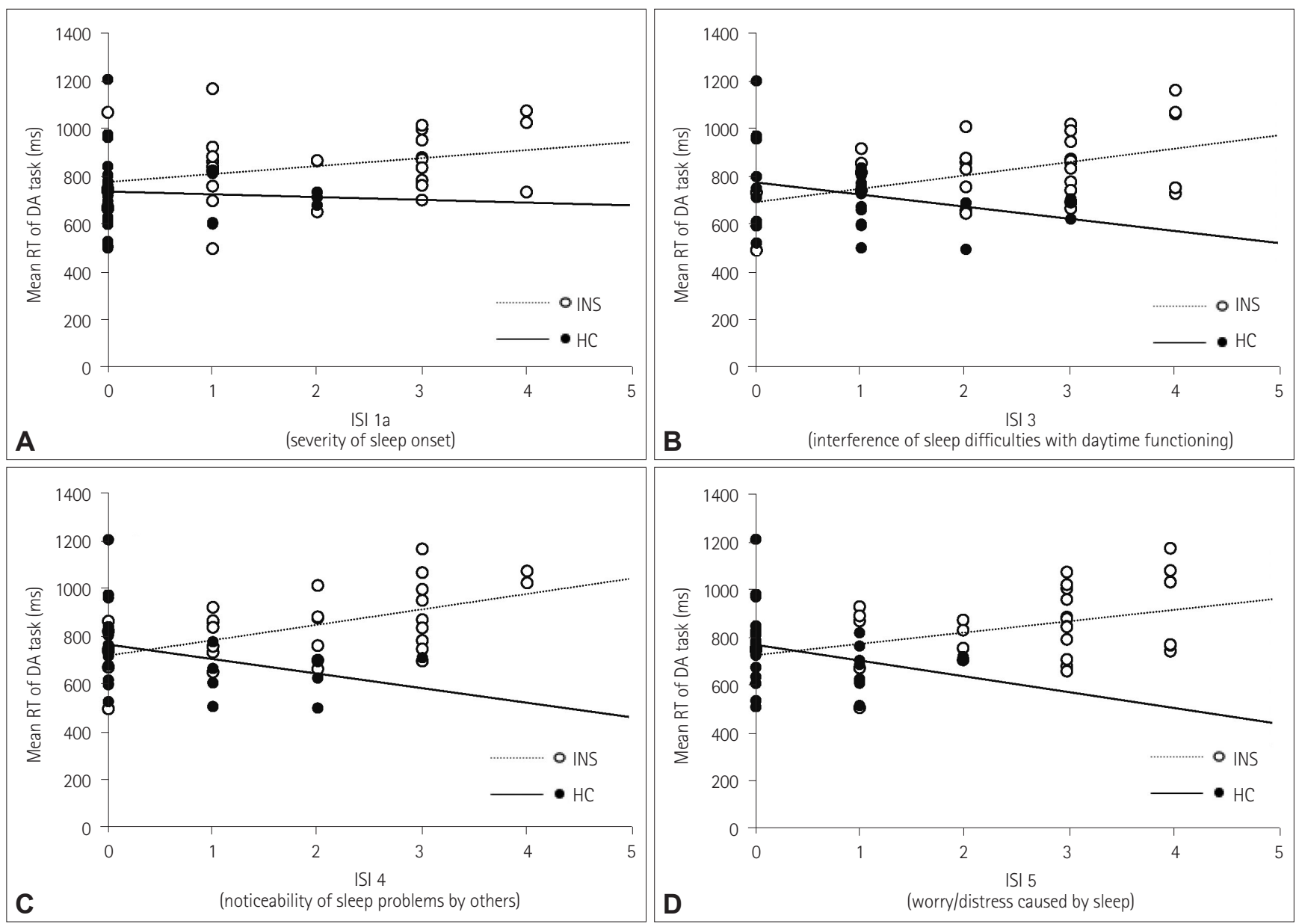

Figure 3. Correlation between DA performance and ISI sub-items. Poor attention performance was related to more severe problems with sleep onset $(A)$ and maladaptive thoughts such as the interference of sleep difficulties in daytime functioning (B), the noticeability of sleep problems by others (C), and worry/distress caused by sleep (D) in insomnia patients. The HC group exhibited the opposite correlation. ISI: Insomnia Severity Index, INS: patients with insomnia disorder, HC: healthy control, RT: reaction time, DA: divided attention.

and significant results for items la (severity of sleep onset), 3 (interference of sleep difficulties in daytime functioning), 4 (noticeability of sleep problems by others), and 5 (worry/distress caused by sleep) (Fig. 3). The HC group showed a trend toward a negative correlation for all items and significant results for items 3 and 5 (Fig. 3). There were no significant correlations between mean RT on the SA task and ISI score in either group.

$B D I$. There were no significant correlations between mean RT and BDI score in either group or for either attention task.

BAI. The INS group showed a positive correlation between mean RT on the DA task and BAI score $(\mathrm{r}=0.39, \mathrm{p}<0.05)$ (Table 2, Fig. 4). The HC group showed a trend toward a negative correlation $(\mathrm{r}=-0.28, \mathrm{p}=0.19)$. There were no significant correlations between mean RT on the SA task and BAI score in either group.

$n P S G$. Results showed several significant correlations between attention performance and the nPSG data (Table 2). In the HC group, the mean RT on the SA task was significantly correlated with sleep efficiency (SE) $(r=0.42, \mathrm{p}<0.05)$ (Fig. 1B), and the mean RT on the DA task was significantly correlated with \% of NREM stage $3(r=0.58, p<0.05)$. In the INS group, mean RT on the DA task was significantly correlated with $\%$ of REM $(r=0.39$, $\mathrm{p}<0.05)$ and $\mathrm{SL}(\mathrm{r}=0.44, \mathrm{p}<0.05)$.

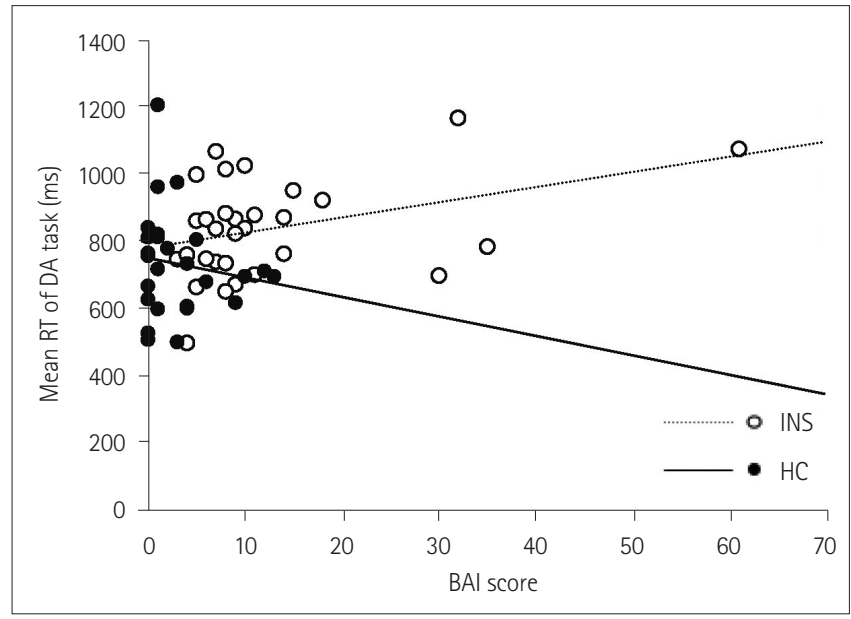

Figure 4. Correlation between DA performance and BAI score. Insomnia patients with higher BAl scores had poorer attention performance. BAI: Beck Anxiety Inventory, INS: patients with insomnia disorder, HC: healthy control, RT: reaction time, DA: divided attention.

\section{DISCUSSION}

The present study aimed to determine whether insomnia patients would differ in attentional function compared with healthy 
controls and to examine the relationships of these differences with the specific characteristics of insomnia. Although negative emotions and maladaptive thoughts are associated with sleep issues, there were no significant differences in attention performance between the INS and HC groups in the present study, consistent with previous findings $[3,4]$. However, there were several trends toward significant differences $(\mathrm{p}=0.06)$. Interestingly, sleep-related symptoms had opposite effects on attention performance in the two groups. Overall, when insomnia patients experienced sleep disturbances or had negative expectations related to sleep loss, their attention performance suffered in a severitydependent manner, with performance becoming poorer as the severity of sleep issues increased. Conversely, when the control group experienced sleep disturbances or had negative effects related to sleep disturbances, they exhibited improved attention performance.

In terms of the specific sleep measures, a high ISI score was associated with poor attention performance in the insomnia patients, particularly with sub-items related to their maladaptive beliefs, including noticeability of sleep problems by others, worry/ distress caused by sleep, and interference of sleep difficulties in daytime functioning. The HC group showed opposite results, which is not surprising. The maladaptive beliefs of insomnia patients are a major factor leading to chronic insomnia, as these beliefs intensify arousal levels to sleep-related stimuli and prompt safety behaviors that generate bad sleep habits, ultimately resulting in repeated disturbance of sleep during the night and dysfunction during the day [10]. This subjective misunderstanding of sleep occurs even when their sleep is not objectively disturbed [18]. This psychological process seems to affect the mindset necessary to maintain attentional effort for functioning well in daily life [19]. The present results confirm that these maladaptive beliefs have a negative effect on maintaining stable attentional efforts.

Of the ISI sub-items assessing various types of sleep disturbance, poor attention performance was related to sleep initiation difficulty in the INS group. Insomnia patients frequently report uncontrolled and unwanted worries or thoughts about sleep in the presleep stage $[20,21]$. These unconstructive worries and ruminations result in psychological and physiological arousal and delay sleep onset $[22,23]$, this arousal state is strongly associated with severe anxiety in insomnia patients [10]. Relatedly, insomnia patients' anxiety scores were correlated with poor attention performance. Unlike other cognitive domains, attentional effort requires heightened and active psychological effort and is thus vulnerable to characteristics exhibited by insomnia patients, including repetitive sleep-related stress and heightened anxiety levels.

In contrast, poor sleep quality (based on PSQI scores) was associated with good attention performance in the HC group, and a similar result was observed regarding SE (as measured by $\mathrm{nPSG}$ ). Many previous studies of sleep deprivation have reported that sleep loss does not affect attention performance and have further suggested that these negative findings may be due to the compensatory recruitment of cognitive engagement following sleep de- privation. For example, one model suggests that sleep deprivation may have a more significant effect on simple or monotonous tasks that require less high-level top-down control relative to complex tasks [24]. Moreover, attention performance is impaired when the task load is increased beyond one's ability to compensate, such as during a triple task compared with a dual task [25], a commonly used paradigm in sleep deprivation studies. However, the present study found that a greater degree of sleep disturbance resulted in better attention performance in the HC group; this may be due to motivation levels. Motivation is required for the maintenance of attentional efforts; it was previously explained as a cognitive incentive for optimizing input processing, detecting errors, and focusing processing resources under challenging circumstances [26]. As mentioned above, following sleep disturbance, attention performance is more vulnerable during tedious and repetitive attention tasks than during complex tasks that require high levels of top-down control. The task used in the present study seems to have triggered sufficient top-down control, and it may be that, in the presence of increased sleep disturbance, highly motivated cognitive engagement was required of the $\mathrm{HC}$ group to enable compensatory resources.

The present study has several limitations that should be considered. First, it was not possible to directly compare the cognitive properties of SA with those of DA. In the HC group, severe sleep disturbances were associated with better performance on both the SA and DA tasks, whereas associations between attention performance and the clinical characteristics of insomnia patients were observed only for the DA task. This finding differs from those of previous studies, which reported that sleep loss was more relevant during simple than during complex attention tasks. Perhaps the 10-min SA task in the present study was at high enough level that insomnia patients were not affected by their clinical symptoms. However, this possibility is only a hypothesis because it was not possible to directly compare performance on the SA task with that on the DA task or to determine why this difference occurred. In the future, it would be valuable to investigate how differing levels of attention might be related to the symptoms of insomnia patients.

Based on the theoretical lapse hypothesis of sleep deprivation, which suggests that sleep deprivation does not completely impair cognitive function but rather aggravates attention dysfunction as a result of an unstable awake state [5], the present study examined attention performance to better understand the daily dysfunction of insomnia patients. The results showed that sleep-related difficulties, such as a lower quality of sleep or worries/distress about sleep, had a positive influence on attention function in the HC group, which likely occurred because these issues motivated them to use compensatory resources. On the other hand, these difficulties had a negative impact on insomnia patients and resulted in poorer attention. These results indicate that the attention complaints of insomnia patients may be associated with a failure to maintain attentional efforts due to sleep-related psychological symptoms or anxiety rather than due to objective cognitive impairments. 


\section{Acknowledgments}

This research was supported by the Brain Research Program through the National Research Foundation of Korea grant funded by the Ministry Science of ICT \& the Ministry Education (Study No. NRF-2015R1C1A2A01054060, NRF-2018R1D1A1B07049704).

\section{Conflicts of Interest}

The authors have no potential conflicts of interest to disclose.

\section{Author Contributions}

Conceptualization: Yu Jin Lee. Data curation: Soohyun Kim, Juhyun Park, Seong min Oh, Mi hyun Lee, Jeong Eun Jeon, Ha Young Lee. Formal analysis: Soohyun Kim. Funding acquisition: Yu Jin Lee. Investigation: Soohyun Kim, Juhyun Park, Seong min Oh, Mi hyun Lee, Jeong Eun Jeon, Ha Young Lee. Project administration: Yu Jin Lee. Resources: Yu Jin Lee. Supervision: Yu Jin Lee. Visualization: Soohyun Kim. Writing—original draft: Soohyun Kim. Writing_-review \& editing: Soohyun Kim, Yu Jin Lee.

\section{ORCID iDs}

Yu Jin Lee (1)

https://orcid.org/0000-0001-5195-2579

Soohyun Kim (1)

https://orcid.org/0000-0003-0424-8575

\section{REFERENCES}

1. Roth T, Ancoli-Israel S. Daytime consequences and correlates of insomnia in the United States: results of the 1991 National Sleep Foundation Survey. II. Sleep 1999;22 Suppl 2:S354-S358.

2. Linton SJ, Bryngelsson L. Insomnia and its relationship to work and health in a working-age population. J Occup Rehabil 2000;10:169-183.

3. Fortier-Brochu E, Beaulieu-Bonneau S, Ivers H, Morin CM. Insomnia and daytime cognitive performance: a meta-analysis. Sleep Med Rev 2012; 16:83-94.

4. Wardle-Pinkston S, Slavish DC, Taylor DJ. Insomnia and cognitive performance: a systematic review and meta-analysis. Sleep Med Rev 2019;48:101205.

5. Dorrian J, Rogers NL, Dinges DF. Psychomotor vigilance performance: neurocognitive assay sensitive to sleep loss. In: Kushida CA, editor. Sleep deprivation. 1st ed. Boca Raton (FL): CRC Press, 2004, p. 39-70.

6. Altena E, Van Der Werf YD, Strijers RL, Van Someren EJ. Sleep loss affects vigilance: effects of chronic insomnia and sleep therapy. J Sleep Res 2008; 17:335-343.

7. Varkevisser M, Kerkhof GA. Chronic insomnia and performance in a 24-h constant routine study. J Sleep Res 2005;14:49-59.

8. Fortier-Brochu E, Morin CM. Cognitive impairment in individuals with insomnia: clinical significance and correlates. Sleep 2014;37:1787-1798.

9. Orff HJ, Drummond SP, Nowakowski S, Perils ML. Discrepancy between subjective symptomatology and objective neuropsychological performance in insomnia. Sleep 2007;30:1205-1211.

10. Harvey AG. A cognitive model of insomnia. Behav Res Ther 2002;40:869-893.

11. Espie CA. Understanding insomnia through cognitive modelling. Sleep Med 2007;8 Suppl 4:S3-S8.

12. Harvey AG. Identifying safety behaviors in insomnia. J Nerv Ment Dis 2002;190:16-21.

13. Harvey AG, Greenall E. Catastrophic worry in primary insomnia. J Behav Ther Exp Psychiatry 2003;34:11-23.

14. Taylor DJ, Lichstein KL, Durrence HH, Reidel BW, Bush AJ. Epidemiology of insomnia, depression, and anxiety. Sleep 2005;28:1457-1464.

15. American Psychiatric Association. Diagnostic and statistical manual of mental disorders: DSM-5. 5th ed. Arlington (VA): American Psychiatric Publishing, Inc.; 2013.

16. Yoo HK, Lee J, Kang SH, Park EH, Jung J, Kim BN, et al. Standardization of the Comprehensive Attention Test for the Korean children and adolescents. J Korean Acad Child Adolesc Psychiatry 2009;20:68-75.

17. Huh HN, Kang SH, Hwang SY, Yoo HK. Developmental trajectories of attention in normal Korean population. J Korean Acad Child Adolesc Psychiatry 2019;30:66-73.

18. Semler CN, Harvey AG. Misperception of sleep can adversely affect daytime functioning in insomnia. Behav Res Ther 2005;43:843-856.

19. Riedel BW, Lichstein KL. Insomnia and daytime functioning. Sleep Med Rev 2000;4:277-298.

20. Harvey AG. Pre-sleep cognitive activity: a comparison of sleep-onset insomniacs and good sleepers. Br J Clin Psychol 2000;39:275-286.

21. Gerlach F, Ehring T, Werner GG, Takano K. Insomnia-related interpretational bias is associated with pre-sleep worry. J Sleep Res 2020;29:e12938.

22. Vandekerckhove M, Weiss R, Schotte C, Exadaktylos V, Haex B, Verbraecken J, et al. The role of presleep negative emotion in sleep physiology. Psychophysiology 2011;48:1738-1744.

23. Pillai V, Drake CL. Sleep and repetitive thought: the role of rumination and worry in sleep disturbance. In: Babson KA, Feldner MT, editors. Sleep and affect. London: Academic Press, 2015, p. 201-225.

24. Pilcher JJ, Band D, Odle-Dusseau HN, Muth ER. Human performance under sustained operations and acute sleep deprivation conditions: toward a model of controlled attention. Aviat Space Environ Med 2007;78(5 Suppl):B15-B24.

25. Chua EC, Fang E, Gooley JJ. Effects of total sleep deprivation on divided attention performance. PLoS One 2017;12:e0187098.

26. Sarter M, Gehring WJ, Kozak R. More attention must be paid: the neurobiology of attentional effort. Brain Res Rev 2006;51:145-160. 